Luiz Gustavo Oliveira Brito ${ }^{1}$

Júlio César Rosa e Silva ${ }^{1}$ ANTONIO Alberto NOGUEIRA

\title{
Reflexões sobre o impacto causado pelo alerta do Food and Drug Administration (FDA) americano sobre o morcelamento eletromecânico uterino e/ou de miomas
}

\section{Reflections about the impact caused by the Food and Drug Administration (FDA) warning against uterine andlor fibroid power morcellation}

A histerectomia por doença benigna ainda é a cirurgia ginecológica mais realizada no mundo, e apesar do avanço dos tratamentos clínicos e minimamente invasivos para diversas causas, como leiomioma uterino, suas taxas se mantêm estáveis, sem mostrar tendência de diminuição nos Estados Unidos ${ }^{1}$. Com o advento das vias minimamente invasivas, crescem no mundo todo as abordagens laparoscópica e robótica, diminuindo o percentual da abordagem laparotômica; isso repercute em benefícios para a paciente, trazendo menos dor, recuperação mais rápida, assim como retorno precoce às atividades habituais ${ }^{2}$.

Em úteros de grande volume, a redução do volume do espécime no intra-operatório com o emprego de morcelamento levou essa possibilidade da via menos invasiva também para essas mulheres cujo útero seria dificilmente extraído por via vaginal. O morcelamento consiste em cortar (com bisturi frio ou roteadores com lâminas) um útero volumoso em pequenos pedaços e retirá-lo pela via vaginal ou através dos trocárteres cirúrgicos no final da cirurgia ${ }^{3}$. De 600 mil histerectomias realizadas anualmente nos Estados Unidos, em torno de 50 a 150 mil são realizadas com o power morcellator ou morcelador eletromecânico. Sua técnica vem sendo utilizada há 20 anos, com altas taxas de sucesso e baixas taxas de complicação. Uma recente revisão da base de dados americana MAUDE, que agrupa complicações relacionadas ao uso de dispositivos, mostrou em uma análise de 10 anos a ocorrência de 10 mortes e 214 eventos associados a lesão intra-operatória $(n=47)$ ou atraso do procedimento devido à falha do power morcellator $(\mathrm{n}=140)^{4}$.

Sabe-se que um baixíssimo risco de leiomiossarcoma incidental (1/350 a 1/1.000) pode ocorrer ${ }^{5}$ pela disseminação de material biológico na cavidade, e teoricamente todo cirurgião ginecológico deveria orientar suas pacientes para essa possibilidade em caso de morcelamento intra-operatório ${ }^{6,7}$.

Esse risco ganhou proporções midiáticas de grande alcance em novembro de 2013, quando ocorreram casos isolados nos Estados Unidos de leiomiossarcomas incidentais durante histerectomias laparoscópicas nas quais que se utilizou o power morcellator para reduzir o volume da peça para extração. Houve uma grande procura sobre o tema "morcelamento uterino" conforme a ferramenta de busca Google Trends ${ }^{8}$ (Figura 1).

Correspondência

Luiz Gustavo Oliveira Brito Avenida Bandeirantes, 3900 - Monte Alegre CEP: $14049-900$

Ribeirão Preto (SP), Brasil

Recebido

$03 / 06 / 2015$

Aceito com modificações

$30 / 06 / 2015$

'Departamento de Ginecologia e Obstetrícia, Faculdade de Medicina de Ribeirão Preto, Universidade de São Paulo - USP - Ribeirão Preto (SP), Brasil.

Conflito de interesses: não há 


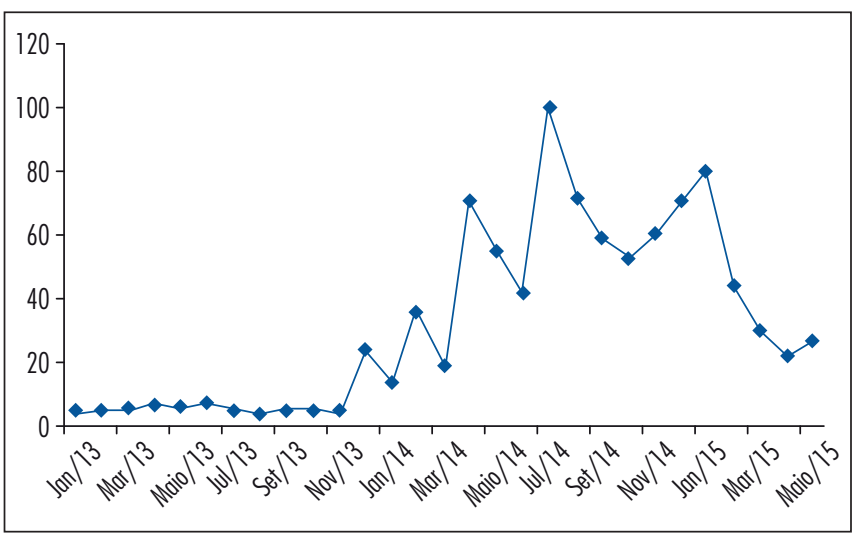

Figura 1. Pesquisa no Google Trends da expressão uterine morcellation. Os dados são normalizados para uma população de referência e escalonados de 0 a $100 \%$, sendo o valor máximo chamado de atividade pico de busca

Especificamente, o primeiro efeito adverso relatado ao Food and Drug Administration (FDA) sobre leiomiossarcoma provindo de uma histerectomia laparoscópica com o power morcellator foi de uma anestesiologista que trabalhava em um hospital em Boston e foi submetida ao procedimento9 . Ela expôs seu caso na mídia e reuniu em torno de aproximadamente 100 mulheres que teriam passado pela mesma situação. Tais casos repercutiram na mídia de forma intensa e fizeram o FDA se manifestar frente a essas denúncias de pacientes. Em abril de 2014, o FDA lançou um alerta ${ }^{10}$ sobre o uso do power morcellator, desencorajando o seu uso durante a laparoscopia. A recomendação para os profissionais de saúde é que o médico exponha todas as opções disponíveis para mulheres com indicação cirúrgica, e que a paciente fique bem orientada sobre o risco de disseminação intracavitária de células tumorais. Uma das grandes indústrias produtoras do power morcellator retirou seus produtos de circulação em julho de $2014^{11}$. Depois do primeiro alerta, uma reunião foi realizada em julho com uma equipe de experts no assunto e em novembro uma atualização desse alerta foi publicado, dessa vez sendo mais incisivo de forma a não recomendar o uso desses dispositivos para mulheres cujo risco para câncer oculto não seja desprezível ou para mulheres na peri ou pós-menopausa cuja histerectomia tenha sido indicada por leiomioma uterino ${ }^{12}$.

O impacto do morcelamento de leiomiossarcomas incidentais tem sido analisado de forma mais frequente na literatura médica, com reforço no número de casuísticas devido aos fatos ocorridos. Uma metanálise de quatro estudos mostrou que o morcelamento aumentou em três vezes a taxa de recorrência geral, quatro vezes a de intra-abdominal e duplicou o risco de morte ${ }^{13}$. Contudo, o nível de viés dos trabalhos selecionados era alto e a amostra insuficiente para concluir de forma precisa tais resultados. Trabalhos com casuísticas isoladas após a publicação dessa revisão sistemática confirmam redução no intervalo livre de doença e sobrevida geral nesse grupo de mulheres ${ }^{14,15}$. O prognóstico é mais atenuado para tumores de músculo liso de potencial maligno incerto (STUMP — smooth muscle tumors of uncertain malignant potential) e sarcoma de estroma endometrial, porém, a reexploração cirúrgica após morcelamento desses tumores apresenta alta probabilidade de detectar implantes peritoneais, sejam benignos ou malignos ${ }^{16}$.

A interrupção do morcelamento uterino tem levado inúmeros cirurgiões ginecológicos a aumentarem o número de incisões para extração do espécime, assim como muitas pacientes a desejarem a via aberta. Orientações médico-legais foram recentemente publicadas visando otimizar o processo de decisão cirúrgica com o paciente ${ }^{17}$. Mas será que o percentual de mortes por leiomiossarcoma incidental por via laparoscópica seria maior do que o percentual de mortes por complicações decorrentes de histerectomias abdominais abertas? Um estudo recém-publicado mostra resultados interessantes empregando um modelo de árvore de decisão com 100 mil mulheres pré-menopausadas que hipoteticamente se submeteriam a uma histerectomia. Embora ocorressem mais mortes por leiomiossarcoma depois de uma histerectomia laparoscópica (86 versus 71/100 mil), houve mais mortes relacionadas a histerectomia na via abdominal (32 versus 12/100 mil), mostrando um equilíbrio entre as duas opções e a necessidade de colocar a paciente como o objeto central no processo de decisão cirúrgica junto ao médico ${ }^{18}$.

Com a polarização das opiniões acerca de realizar ou não o morcelamento, novas técnicas têm sido estimuladas para realizar o morcelamento de forma segura e eficaz. É importante lembrar que nenhuma delas foi padronizada pelo FDA ou outro órgão regulamentador, sendo estudos-piloto na maioria dos casos. A utilização de sacos com material flexível e que seja resistente à distensão por pneumoperitônio tem sido avaliada em estudos prospectivos; tal técnica tem sido chamada de in-bag morcellation (IBM). Um estudo com um grupo prospectivo, piloto com 36 mulheres para as quais se empregou o IBM e um retrospectivo das últimas 49 pacientes tratadas com morcelamento com o power morcellator mostrou um aumento de 20 minutos no tempo cirúrgico, sem diferenças quanto a perda sanguínea, peso do espécime, tempo de internação e complicações. Não houve roturas no saco utilizado no procedimento ${ }^{19}$. Outro estudo prospectivo de 73 pacientes não mostrou danos ao saco utilizado, nem perda de material na cavidade peritoneal durante o procedimento ${ }^{20}$. Vídeos sobre essa técnica têm sido elaborados ${ }^{21}$. Essa técnica representa uma possibilidade para as histerectomias supracervicais ou miomectomias. 
O morcelamento via vaginal também tem sido estudado, em menor escala, tanto para doenças benignas ${ }^{22}$ quanto para malignas ${ }^{23}$, apresentando bons resultados. Ainda não existem dados sobre o melhor material para envolver o espécime, assim como a definição da melhor via para realizar o procedimento. É importante lembrar que tais técnicas são experimentais e ligadas a um protocolo de pesquisa; portanto, em caso de desejo de aplicação, um projeto deve ser elaborado e aprovado pela instituição responsável.

Espera-se uma nova definição do FDA sobre o tema. Por enquanto, se não houver condições seguras de se realizar o procedimento, se o morcelamento não puder ser realizado em ambiente seguro (ex. utilização de bolsas), a minilaparotomia e/ou a extração vaginal para o espécime são as opções de escolha para a paciente.

\section{Referências}

1. Wright JD, Herzog TJ, Tsui J, Ananth CV, Lewin SN, Lu YS, et al. Nationwide trends in the performance of inpatient hysterectomy in the United States. Obstet Gynecol. 2013;122(2 Pt 1):233-41.

2. Lee J, Jennings K, Borahay MA, Rodriguez AM, Kilic GS, Snyder $R R$, et al. Trends in the national distribution of laparoscopic hysterectomies from 2003 to 2010. J Minim Invasive Gynecol. 2014;21(4):656-61.

3. Bogani G, Uccella S, Cromi A, Serati M, Casarin J, Sturla D, et al. Electric motorized morcellator versus transvaginal extraction for myoma retrieval after laparoscopic myomectomy: a propensitymatched analysis. J Minim Invasive Gynecol. 2014;21 (5):928-34.

4. Naumann RW, Brown J. Complications of electromechanical morcellation reported in the MAUDE Database. J Minim Invasive Gynecol. 2015 May 15. [Epub ahead of print]

5. Parker WH, Fu YS, Berek JS. Uterine sarcoma in patients operated on for presumed leiomyoma and rapidly growing leiomyoma. Obstet Gynecol. 1994;83(3):414-8

6. Park JY, Park SK, Kim DY, Kim JH, Kim YM, Kim YT, et al. The impact of tumor morcellation during surgery on the prognosis of patients with apparently early uterine leiomyosarcoma. Gynecol Oncol. $2011 ; 122(2): 255-9$.

7. George S, Barysauskas C, Serrano C, Oduyebo T, Rauh-Hain JA, Del Carmen MG, et al. Retrospective cohort study evaluating the impact of intraperitoneal morcellation on outcomes of localized uterine leiomyosarcoma. Cancer. 2014;120(20):3154-8.

8. Brito LG, Ferriani RA, Candido-dos-Reis FJ, Nogueira AA. Using a search-volume tool (Google Trends) to assess global interest for uterine fibroids. Arch Gynecol Obstet. 2014;289(6): 1 163-4.

9. Brower V. FDA considers restricting or banning laparoscopic morcellation. J Natl Cancer Inst. 2014;106(10):2-3.

10. U.S. Food and Drug Administration (FDA). Laparoscopic uterine power morcellation in hysterectomy and myomectomy: FDA safety communication [Internet]. Geneva: WHO; 2014 [cited 2015 May 5]. Available from: <http://www.fda.gov/MedicalDevices/Safery/ AlertsandNotices/ucm393576.htm>

11. Kamp J, Levitz J. Johnson \& Johnson pulls hysterectomy device from hospitals. [Internet]. Wall Street Journal. 2014 Jul 30 [cited 2015 May 5]. Available from: <http://www.wsj.com/articles/johnsonjohnson-to-call-for-voluntary-return-of-morcellators-1406754350>

12. Food and Drug Administration (FDA). Updated laparoscopic uterine power morcellation in hysterectomy and myomectomy: FDA safety communication. [Internet]. Geneva: WHO; 2014 [cited 2015
Jun 6]. Available from: <http://www.fda.gov/MedicalDevices/ Safety/AlertsandNotices/ucm424443.htm>

13. Bogani G, Cliby WA, Aletti GD. Impact of morcellation on survival outcomes of patients with unexpected uterine leiomyosarcoma: a systematic review and meta-analysis. Gynecol Oncol. $2015 ; 137(1): 167-72$.

14. Lin $\mathrm{KH}$, Torng PL, Tsai KH, Shih HJ, Chen CL. Clinical outcome affected by tumor morcellation in unexpected early uterine leiomyosarcoma. Taiwan J Obstet Gynecol. 2015;54(2):172-7.

15. Bojahr B, De Wilde RL, Tchartchian G. Malignancy rate of 10,731 uteri morcellated during laparoscopic supracervical hysterectomy (LASH). Arch Gynecol Obstet. 2015 Mar 28. [Epub ahead of print]

16. Mowers EL, Skinner B, McLean K, Reynolds RK. Effects of morcellation of uterine smooth muscle tumor of uncertain malignant potential and endometrial stromal sarcoma: case series and recommendations for clinical practice. J Minim Invasive Gynecol. 2015;22(4):601-6.

17. Ton R, Kilic GS, Phelps JY. A medical-legal review of power morcellation in the face of the recent FDA warning and litigation. J Minim Invasive Gynecol. 2015;22(4):564-72.

18. Siedhoff MT, Wheeler SB, Rutstein SE, Geller EJ, Doll KM, Wu JM, et al. Laparoscopic hysterectomy with morcellation vs abdominal hysterectomy for presumed fibroid tumors in premenopausal women: a decision analysis. Am J Obstet Gynecol. 2015;212(5):591 .e 1-8.

19. Vargas MV, Cohen SL, Fuchs-Weizman N, Wang KC, Manoucheri E, Vitonis $A F$, et al. Open power morcellation versus contained power morcellation within an insufflated isolation bag: comparison of perioperative outcomes. J Minim Invasive Gynecol. $2015 ; 22(3): 433-8$.

20. Cohen SL, Einarsson Jl, Wang KC, Brown D, Boruta D, Scheib SA, et al. Contained power morcellation within an insufflated isolation bag. Obstet Gynecol. 2014;124(3):491-7.

21. McKenna JB, Kanade T, Choi S, Tsai BP, Rosen DM, Cario GM, et al. The Sydney contained in bag morcellation technique. J Minim Invasive Gynecol. 2014;21(6):984-5.

22. Günthert AR, Christmann C, Kostov P, Mueller MD. Safe vaginal uterine morcellation following total laparoscopic hysterectomy. Am J Obstet Gynecol. 2015;212(4):546.e 1-4.

23. Favero G, Miglino G, Köhler C, Pfiffer T, Silva e Silva A, Ribeiro $A$, et al. Vaginal morcellation inside protective pouch: a safe strategy for uterine extration in cases of bulky endometrial cancers: operative and oncological safety of the method. J Minim Invasive Gynecol. 2015 Apr 24. [Epub ahead of print] 